\title{
Double Coded Genomic Data and Samples
}

\author{
National Cancer Institute
}

\section{Source}

National Cancer Institute. Double Coded Genomic Data and Samples. NCI Thesaurus. Code C68758.

Double coded data and samples are initially labelled with a single code and do not carry any personal identifiers. The data and samples are then relabelled with a second code, which is linked to the first code via a second coding key. It is possible to trace the data or samples back to the individual by the use of both coding keys. In general, the clinical investigator is responsible for maintaining the first coding key and does not have access to the second coding key. As the samples and associated data are indirectly traceable back to the subject via the use of both coding keys, it is possible to undertake actions such as sample withdrawal, or the return of individual results. The use of double coded data and samples allows for clinical monitoring, subject follow-up or the addition of new data. The use of the second code provides additional confidentiality and privacy protection for subjects over that of single coded. Access to both coding keys is needed to link any data or samples back to a subject identifier (ICH). 